\title{
Validation of Spectroscopic Model for Fe Ions in Non-Equilibrium Ionization Plasma in LHD and Hinode ${ }^{*}$
}

\author{
Izumi MURAKAMI ${ }^{1,2)}$, Tetsuya WATANABE ${ }^{3,4)}$, Chihiro SUZUKI ${ }^{1)}$, Shigeru MORITA ${ }^{1,2)}$, \\ Chunfeng DONG ${ }^{1)}$, Naoki TAMURA ${ }^{1)}$, Norimasa YAMAMOTO ${ }^{5)}$, Daiji KATO ${ }^{1,2)}$, \\ Hiroyuki A. SAKAUE ${ }^{1)}$, Hirohisa HARA ${ }^{3,4)}$, Nobuyuki NAKAMURA ${ }^{6)}$ \\ and Shigeru SUDO ${ }^{1,2)}$ \\ ${ }^{1)}$ National Institute for Fusion Science, Toki, Gifu 509-5292, Japan \\ ${ }^{2)}$ Department of Fusion Science, The Graduate University of Advanced Studies (SOKENDAI), Toki, Gifu 509-5292, Japan \\ ${ }^{3)}$ National Astronomical Observatory of Japan, Mitaka, Tokyo 181-8588, Japan \\ ${ }^{4)}$ Department of Astronomical Science, The Graduate University of Advanced Studies (SOKENDAI), Mitaka, Tokyo \\ 181-8588, Japan \\ ${ }^{5)}$ Center of Applied Superconductivity and Sustainable Energy Research, Chubu University, Kasugai, Aichi \\ 487-8501, Japan \\ ${ }^{6}$ Institute for Laser Science, The University of Electro-Communications, Chofu, Tokyo 182-8585, Japan
}

(Received 25 September 2013 / Accepted 16 February 2014)

\begin{abstract}
We measured extreme ultraviolet spectra of Fe ions for plasmas produced in the Large Helical Device (LHD) at the National Institute for Fusion Science (NIFS). Iron was injected into the plasmas by using a tracerencapsulated pellet. By controlling the neutral beam injection pattern, we could produce plasma with a central electron temperature of approximately $500 \mathrm{eV}$, which was suitable for producing Fe XVII ions. We measured seven Fe XVII lines. The intensity ratio for $\lambda$ of 20.468 to $25.493 \mathrm{~nm}$ was consistent with the theoretically calculated value of 1.1. This calculated value was determined purely from the branching ratio due to the common upper level of these transitions, although Warren et al. [Astrphys. J. 685, 1277 (2008)] reported a larger ratio of approxinately 2 from Hinode EIS measurements. The other five ratios for Fe XVII lines in our LHD measurements were also consistent with the theoretical ratios calculated with a collisional-radiative model. A preferred atomic dataset for Fe XVII is suggested to obtain better agreement between the measured and calculated ratios.
\end{abstract}

(c) 2014 The Japan Society of Plasma Science and Nuclear Fusion Research

Keywords: Fe XVII spectral lines, spectroscopic diagnostics, EUV spectrum, solar plasma, fusion plasma

DOI: $10.1585 /$ pfr.9.1401056

\section{Introduction}

In solar physics, one of the major issues to be solved is the identification of the heating mechanism that keeps the hot and diffuse corona, extending above the Sun's cool atmosphere at its high temperature of a few million degrees. Since 2006, the Hinode spacecraft has been in orbit to study the heating mechanism and dynamics of the active solar corona, the origin of strong magnetic fields, and the trigger mechanism for solar flares [1]. Hinode is equipped with a solar optical telescope (SOT), an X-ray telescope (XRT), and an extreme ultraviolet (EUV) imaging spectrometer (EIS). The EIS takes EUV images of the Sun as well as EUV spectra in the wavelength regions of $17.0-21.0 \mathrm{~nm}$ and $25.0-29.0 \mathrm{~nm}$ [2]. In these regions, $\mathrm{Fe}$ VIII-XXIV lines are measurable and are expected to provide information on the solar plasma in the wide electron temperature range $4.7<\log T_{\mathrm{e}}[\mathrm{K}]<7.2$.

author'se-mail: murakami.izumi@nifs.ac.jp

*) This article is based on the invited talk at the 29th JSPF Annual Meeting (2012, Fukuoka).
Several models have been proposed to explain coronal heating by acoustic waves, Alfvén body waves, microflares, etc [3]. Conventional one-fluid MHD plasma in thermal equilibrium has been used for modeling solar plasma. Typical ions observed in the solar transition region and the corona have long ionization and recombination timescales, and non-equilibrium ionization has been suggested especially for plasma with flow [4] or solar flares [5]. When the ionization timescale is longer than the time required for an ion to traverse a temperature scale height, the degree of ionization is delayed behind the equilibrium value under local conditions. In the solar transition region, the electron temperature and density change drastically, and non-equilibrium conditions can result from downflows or outflows in the transition region. Dupree et al. [4] showed that the electron temperature dependences of O IV, N IV, and C IV spectral-line intensity ratios are altered when plasma is in non-equilibrium with outflows in the transition region. Imada et al. [6] found that the $\mathrm{Fe}$ ion state distribution is quite different from that for ion- 
ization equilibrium when time-dependent ionization and recombination processes are considered in the shock region caused by magnetic reconnection. Thus, when analyzing the spectroscopic data, we need to focus on physical conditions of plasma, such as flows and shocks. In addition, non-thermal velocities measured as an excess of line thermal broadening are thought to be caused by turbulence, waves, or velocity gradients; those velocities provide important information for examining the coronal heating mechanism [7]. Doscheck et al. [7] and Imada et al. [8] estimated non-thermal velocities in the solar active region by using Fe XII and Fe XVI lines, and obtained values of $30-60 \mathrm{~km} \mathrm{~s}^{-1}$ and approximately $13 \mathrm{~km} \mathrm{~s}^{-1}$, respectively. Such estimates depend on the accuracy of thermal condition determination for plasmas.

The accuracy of the analyzed results from spectroscopic measurements depends on that of the spectroscopic model and the atomic data used in the model. As Hinode EIS measures Fe VIII-XXIV lines for plasma diagnostics as good indices for the solar transition region over a wide electron temperature range, we have constructed collisional-radiative (CR) models for $\mathrm{Fe}^{7+}-\mathrm{Fe}^{23+}$ ions to analyze both solar and laboratory plasmas [9-14]. We have carefully examined atomic data of $\mathrm{Fe}$ ions to be used in the CR models [15, 16], and so far, have applied the models to the measurements of laboratory plasmas to validate the CR models and atomic data for Fe XIII [9, 14], Fe XIV [14], Fe XV [14], Fe XXI [12], and Fe XXII [13].

The Large Helical Device (LHD) in the National Institute for Fusion Science (NIFS) was used to validate the $\mathrm{CR}$ models and atomic data of Fe ions. The LHD can maintain stable plasmas even after impurity elements are injected by an impurity pellet or a tracer-encapsulated pellet (TESPEL) $[17,18]$. The LHD has various diagnostic systems for measuring electron temperature, density, and other plasma properties. LHD plasmas have electron temperature distributions from a few electron volts in the peripheral region to approximately $4 \mathrm{keV}$ in the plasma center. We can produce plasmas in the LHD with electron temperatures similar to those in the solar transition region and the corona. Thus, we used a TESPEL to inject iron into LHD plasmas and measured EUV spectra to compare with the model calculations $[9,12,13]$. The CR model for Fe XIII evaluated using the LHD experiments [9] has been applied to analyze solar spectra measured by Hinode EIS to estimate the electron density distribution in the solar active region [11].

We also used an electron beam ion trap (EBIT) device and compact EBITs (CoBITs) at the University of ElectroCommunications (UEC) and NIFS [19, 20]. An EBIT ionizes ions by an electron beam and traps them with an electric field of the electron beam. It produces diffuse plasma with an electron density of approximately $10^{10} \mathrm{~cm}^{-3}$. It controls the ion charge distribution by choosing the electron beam energy, and the charge states of spectral lines can be identified easily as only ions with ionization po- tentials smaller than the electron beam energy are produced. EUV spectral lines of Fe XIII, Fe XIV, and Fe XV were measured by the CoBIT at the UEC and compared with the CR model calculations [14]. Electron density dependence of line ratios for Fe XIII and Fe XIV agreed very well with the CR model calculations, but the Fe XV $23.39 \mathrm{~nm} / 24.38 \mathrm{~nm}$ ratio was a factor 1.5 - 2 larger than that obtained from the model calculation. To find the cause of this discrepancy, we need to examine measurements, atomic data, and the CR model even more carefully.

Here we focus on Fe XVII. Recently, Fe XVII spectral lines were measured by Hinode EIS and discrepancies from theoretical predictions were reported for the intensity ratios of Fe XVII $20.4675 \mathrm{~nm}\left(2 \mathrm{p}^{5} 3 \mathrm{~s}^{1} \mathrm{P}_{1}-2 \mathrm{p}^{5} 3 \mathrm{p}^{1} \mathrm{~S}_{0}\right)$ and $25.4885 \mathrm{~nm}\left(2 \mathrm{p}^{5} 3 \mathrm{~s}^{3} \mathrm{P}_{1}-2 \mathrm{p}^{5} 3 \mathrm{p}{ }^{1} \mathrm{~S}_{0}\right)$ [21]. The upper levels of both transitions are the same $\left(2 \mathrm{p}^{5} 3 \mathrm{p}^{1} \mathrm{~S}_{0}\right)$, so the intensity ratio is determined by the branching ratio of 1.1 and does not depend on plasma conditions for optically thin plasma. However, the measured ratio was 2.5. In addition, other Hinode EIS results for this line ratio for the solar active region also show this discrepancy; the ratio tends to increase with decreasing electron temperature [22]. The same lines were measured previously in solar-flare spectra by Skylab and the ratio was 1.1, which agreed with the theoretical prediction [23]. Del Zanna and Ishikawa [24] made a list of Fe XVII EUV lines based on two Hinode EIS measurements, and also reported a discrepancy with respect to theoretical calculations for this line ratio. They suggested that the 20.466-nm line was blended with an unidentified line at $20.472 \mathrm{~nm}$, with the same morphology as the Fe VIII line in the monochromatic images taken by Hinode EIS.

In order to use the Fe XVII lines for diagnosing hightemperature solar active region, the validation of the atomic data for Fe XVII is needed. Jönsson et al. [26] performed theoretical calculations for the Fe XVII atomic structure by using the GRASP2K code, which is widely used for theoretical studies of atoms and is believed to have a high accuracy [25]. Relativistic effects were carefully considered in configuration interaction calculations. The results obtained for the branching ratio of the two Fe XVII lines agreed with the previous prediction [26].

In this study, we used LHD plasmas to measure the Fe XVII spectral lines for validation of the atomic data and the CR model for Fe XVII experimentally and to investigate the discrepancy in the Fe XVII line ratio between theoretical predictions and Hinode EIS measurements. These two Fe XVII lines are very weak and were not detected using CoBIT. In Sec. 2, we briefly introduce the CR model, followed by an explanation of the measurements with the LHD and the analysis in Sec. 3. In Secs. 4 and 5, we discuss the results of validation of the CR model and Hinode EIS measurements. This study is summarized in Sec. 6. 


\section{Collisional-Radiative Model}

The spectral line intensity of the transition from level $i$ to level $j$ is proportional to the product of the population density of the upper level; radiative transition probability; and energy difference of the transition, $n(i) A_{\mathrm{r}}(i, j) \Delta E(i, j)$. We constructed a CR model to calculate the population densities of excited states for an Fe ion using the quasisteady-state approximation. We assumed that population densities of excited states relax fast enough, compared to the timescales for changes in plasma properties and ion densities, and solved the rate equations for population densities as $\mathrm{d} n(i) / \mathrm{d} t=0$. Excited states up to the principal quantum number $n=5$ were considered, and a total of 157 fine-structure levels were included. The rate equation is written as

$$
\begin{aligned}
\frac{\mathrm{d} n(i)}{\mathrm{d} t} & \\
= & \sum_{j \neq i}\left\{C(j, i) n_{\mathrm{e}}+C_{\mathrm{p}}(j, i) n_{\mathrm{p}}\right\} n(j)+\sum_{j>i} A_{\mathrm{r}}(j, i) n(j) \\
& -\sum_{j \neq i}\left\{C(i, j) n_{\mathrm{e}}+C_{\mathrm{p}}(i, j) n_{\mathrm{p}}+S(i) n_{\mathrm{e}}\right\} n(i) \\
& -\sum_{j<i} A_{\mathrm{r}}(i, j) n(i) .
\end{aligned}
$$

We included radiative decay $\left(A_{\mathrm{r}}(i, j)\right)$, electron-impact excitation and de-excitation $(C(i, j))$, electron-impact ionization $(S(i))$, and proton-impact excitation and de-excitation $\left(C_{\mathrm{p}}(i, j)\right)$ processes. Recombination processes were not included in this model because LHD plasmas are mostly in an ionizing plasma phase, and in such cases, recombination processes are not important for the spectral analysis. Energy levels, transition probabilities, and electron-impact excitation and ionization cross sections were calculated with the HULLAC atomic code [27]. Proton-impact rate coefficients were taken from recommended data [15].

For comparison, we also calculated population densities and photon emissivity coefficients using the ADAS package [28]. ADAS contains various atomic datasets for one ion; for comparison, we selected three different atomic datasets for energy levels, transition probabilities, and electron-impact excitation effective collision strengths. Specifically, the selected datasets were "chv6" whose atomic data are taken from CHIANTI ver.6 (245 levels) [29], "cpb06" whose excitation effective collision strengths are obtained by the Dirac-Fock R-matrix method (139 levels) [30], and "lgy09" whose excitation effective collision strengths are obtained by the ICFT Rmatrix method (209 levels) [31]. In the CHIANTI dataset, electron-impact excitation rate coefficients are calculated by the distorted wave method and are included only for certain selected transitions. The same atomic data of "cpb06" were used for the intensity calculations in [24].

\section{LHD Experiments}

EUV spectra in the $10-30 \mathrm{~nm}$ wavelength range were measured for plasmas in LHD using a flat-field EUV spectrometer [32]. The spectrometer can cover a wavelength range of 5-50 nm using a laminar-type holographic grating. Spectral resolution of approximately $0.02 \mathrm{~nm}$ was achieved at $20 \mathrm{~nm}$. A $0.15 \mu \mathrm{m}$ thick aluminum filter was placed in front of the grating to screen out emissions in wavelengths below approximately $17 \mathrm{~nm}$. This successfully reduced the contamination of higher-order emission lines, especially the second-order emission line of Fe XXII at $10.22 \mathrm{~nm}$, which could overlap with the Fe XVII line at $20.468 \mathrm{~nm}$. The exposure time of one frame was $5 \mathrm{~ms}$. A new technique for absolute intensity calibration was conducted using bremsstrahlung continuum radiation and a branching ratio of C IV lines [33].

We analyzed the spectra measured for the plasma of shot number 107802. Figure 1 shows the temporal distribution of the discharge. Three tangential neutral beams (NBIs) were injected at $t=3.3-4.3 \mathrm{~s}$, and two perpendicular neutral beams were injected at $t=4.2-6.2 \mathrm{~s}$. Due to these changes, the stored energy $W_{\mathrm{p}}$ and the central electron temperature started decreasing drastically at $t=4.3 \mathrm{~s}$, reached their minimum at around $t=5 \mathrm{~s}$, and increased again. The minimum central electron temperature was

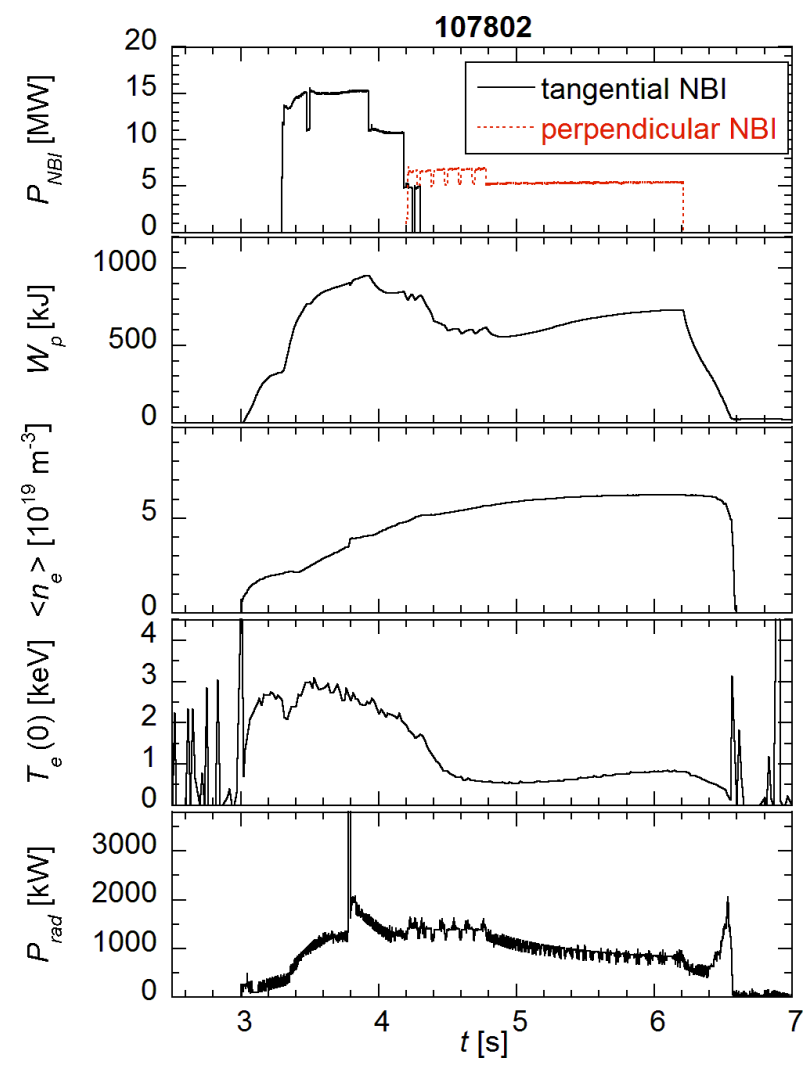

Fig. 1 Temporal evolution of shot 107802 discharge for NBI power $P_{\mathrm{NBI}}$, stored energy $W_{\mathrm{p}}$, line-averaged electron density $\left\langle n_{\mathrm{e}}\right\rangle$, electron temperature at geometrical center $T_{\mathrm{e}}(0)$, and total radiated power $P_{\text {rad }}$. 


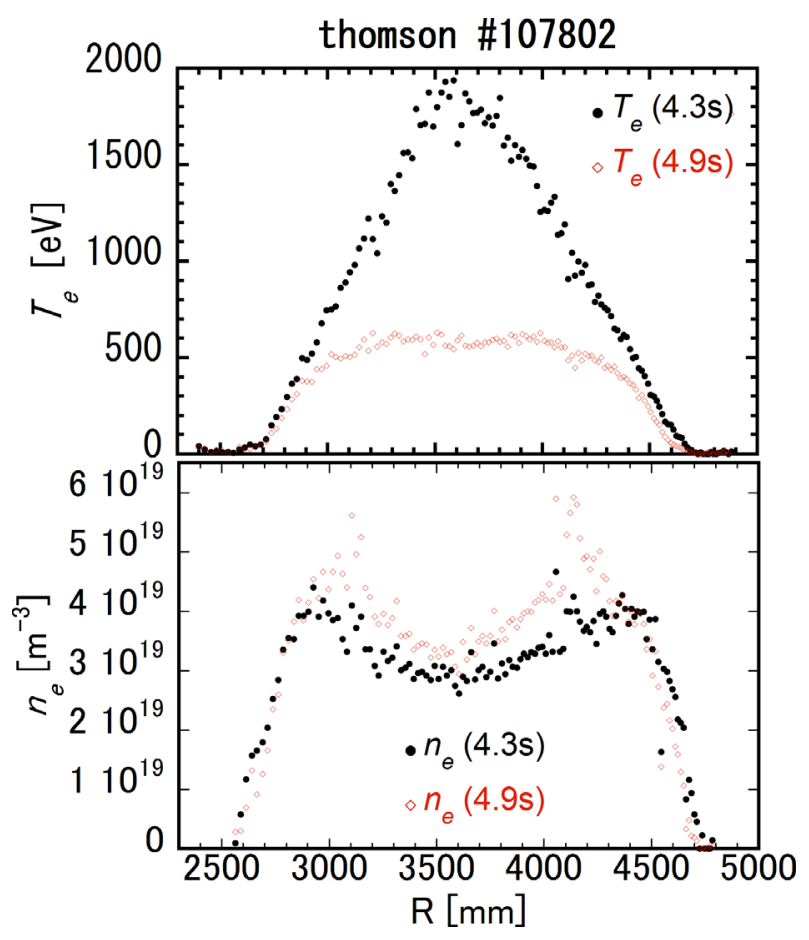

Fig. 2 Electron temperature distributions (top) and electron density distributions (bottom) measured by Thomson scattering at $t=4.3 \mathrm{~s}$ (solid circles) and $4.9 \mathrm{~s}$ (open diamonds).

about $500 \mathrm{eV}$. Figure 2 shows the electron temperature and density distributions at $t=4.3$ and $4.9 \mathrm{~s}$. At $t=4.3 \mathrm{~s}$, the central electron temperature was about $2 \mathrm{keV}$ with a peaky distribution; at $t=4.9 \mathrm{~s}$, the distribution flattened with a central electron temperature of approximately $500 \mathrm{eV}$. This temperature is suitable for Fe XVII measurements. At $t=3.8 \mathrm{~s}$, a TESPEL doped with iron powder was injected. This instantly caused a large radiation power loss, but soon the loss became less effective. The electron temperature and density in the outer region were not affected by the changes in the central region.

Figures 3 (a) and 3 (b) show the EUV spectra at $t=4.4$ and $5.0 \mathrm{~s}$ for wavelength regions $18-22 \mathrm{~nm}$ and $25-29 \mathrm{~nm}$, respectively. At $t=4.4 \mathrm{~s}$, the central electron temperature was high, as shown in Fig. 2, and Fe ions in higher charge states were observed, such as Fe XXIII and Fe XXIV. However, when the central electron temperature decreased to approximately $500 \mathrm{eV}$ at around $t=5.0 \mathrm{~s}$, the higher charged ions disappeared and Fe XVII at 20.468 and $25.493 \mathrm{~nm}$ were clearly detected. Lower charged $\mathrm{Fe}$ ions such as Fe XII and Fe XIII were constantly observed as these ions exist in the peripheral region at lower electron temperatures of approximately $100 \mathrm{eV}$.

As listed in Table 1, a total of seven Fe XVII lines appear in Fig. 3. The measured wavelengths were calibrated with known prominent Fe XXIV lines and other impurity lines. Errors for the line wavelengths measured in the LHD in Table 1 were obtained by fitting to a Gaussian profile. The pixel size of the measurements corresponds to about
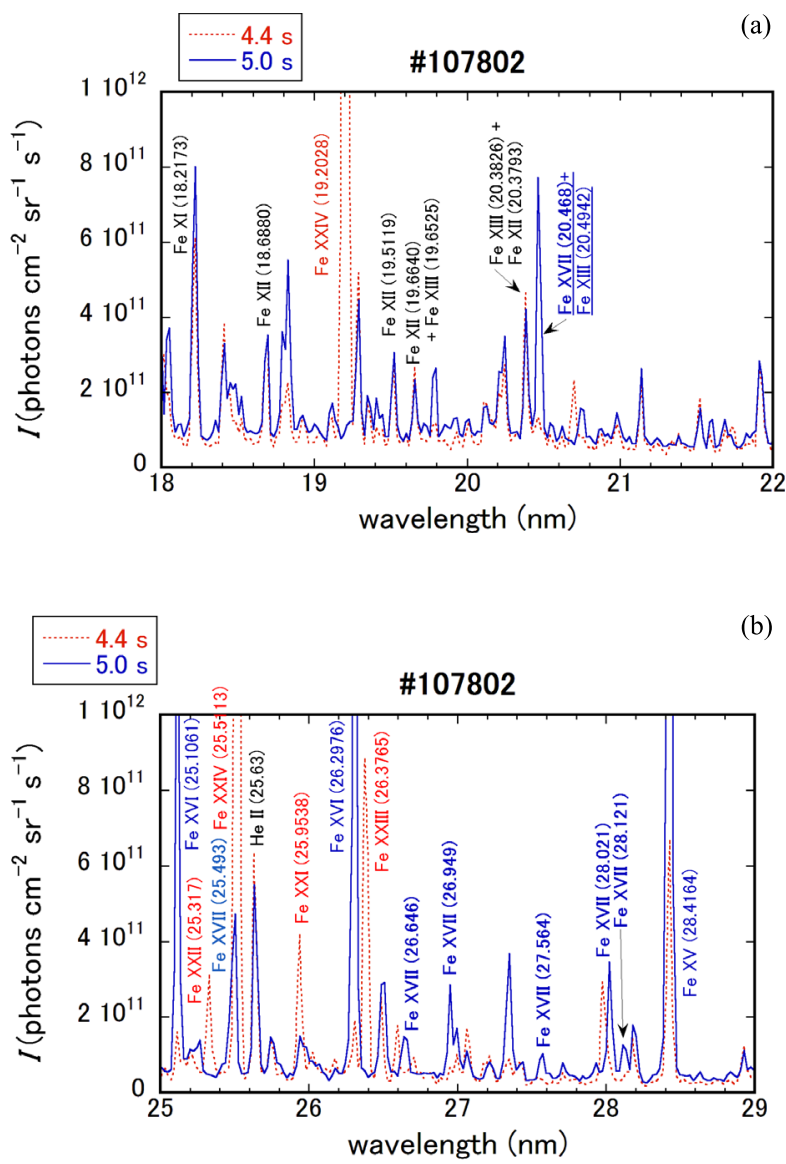

Fig. 3 EUV spectra at $4.4 \mathrm{~s}$ (dotted line) and $5.0 \mathrm{~s}$ (solid line) (a) for $18-22 \mathrm{~nm}$ wavelength region and (b) for $25-29 \mathrm{~nm}$ wavelength region of shot 107802 . Forty frames $(\Delta t=$ $200 \mathrm{~ms}$ ) were added to obtaine the spectra. Wavelengths shown in bold face for identified lines were measured; others were taken from the NIST ASD database [35].

$0.02 \mathrm{~nm}$, but high $\mathrm{S} / \mathrm{N}$ ratios in the measurements allow smaller errors for the line fits. The wavelengths of all $\mathrm{Fe}$ XVII lines roughly agree with those measured by Hinode EIS, those in the NIST database [34], and those calculated by the GRASP code [26]. We follow the identifications of the transitions for the $\lambda 26.646-\mathrm{nm}$ and 27.564-nm lines in the NIST database and the GRASP calculation. The identifications of these two lines are exchanged in the list of Warren et al. [21]. The upper levels of these transitions are mixed with ${ }^{1} D_{2}$ and ${ }^{3} F_{2}$ levels due to configuration mixing, and similarly, the lower levels are mixed with ${ }^{1} \mathrm{P}_{1}$ and ${ }^{3} \mathrm{D}_{1}$ levels.

The lower level of the transition for $\lambda$ of $28.021 \mathrm{~nm}$ (no.6 in Table 1) is mixed with $2 \mathrm{~s}^{2} 2 \mathrm{p}^{5} 3 \mathrm{p}^{1} \mathrm{D}_{2}$ and ${ }^{3} \mathrm{D}_{2}$ levels due to configuration interactions. The term identification of this level in the NIST database [34] differs from that in the GRASP calculation [26], but because the mixing coefficients are almost the same, the levels in the NIST database and the GRASP calculation are expected to be physically identical. The energy levels calculated with the HULLAC code used in our CR model are described with $\mathrm{jj}$ 
Table 1 Observed Fe XVII lines.

\begin{tabular}{|c|c|c|c|c|c|c|}
\hline \multirow[t]{2}{*}{ No. } & \multirow[t]{2}{*}{ Ion } & \multicolumn{2}{|c|}{ Wavelength (nm) } & \multirow[b]{2}{*}{$\mathrm{NIST}^{\mathrm{b}}$} & \multirow[b]{2}{*}{ GRASP $^{c}$} & \multirow{2}{*}{$\begin{array}{c}\text { Transition } \\
\text { Lower level - Upper level }\end{array}$} \\
\hline & & LHD & $\mathrm{EIS}^{\mathrm{a}}$ & & & \\
\hline 1 & Fe XVII & $20.468 \pm 0.001^{\mathrm{d}}$ & 20.4688 & 20.46 & 20.4442 & $2 \mathrm{~s}^{2} 2 \mathrm{p}^{5} 3 \mathrm{~s}^{1} \mathrm{P}_{1}-2 \mathrm{~s}^{2} 2 \mathrm{p}^{5} 3 \mathrm{p}^{1} \mathrm{~S}_{0}$ \\
\hline 2 & Fe XVII & $25.493 \pm 0.002$ & 25.4885 & 25.475 & 25.4471 & $2 s^{2} 2 p^{5} 3 s^{3} P_{1}-2 s^{2} 2 p^{5} 3 p^{1} S_{0}$ \\
\hline 3 & Fe XVII & $26.646 \pm 0.001$ & 26.6417 & 26.643 & 26.6613 & $2 s^{2} 2 p^{5} 3 p^{3} D_{1}-2 s^{2} 2 p^{5} 3 d^{3} F_{2}$ \\
\hline 4 & Fe XVII & $26.949 \pm 0.003$ & 26.9420 & 26.941 & 26.9600 & $2 s^{2} 2 p^{5} 3 p^{3} D_{2}-2 s^{2} 2 p^{5} 3 d^{3} F_{3}$ \\
\hline 5 & Fe XVII & $27.564 \pm 0.007$ & 27.5550 & 27.56 & 27.5774 & $2 s^{2} 2 p^{5} 3 p^{1} P_{1}-2 s^{2} 2 p^{5} 3 d^{1} D_{2}$ \\
\hline \multirow[t]{2}{*}{6} & Fe XVII & $28.021 \pm 0.002$ & 28.0160 & \multicolumn{2}{|l|}{$28.02^{\mathrm{e}}$} & $2 s^{2} 2 p^{5} 3 p^{1} D_{2}-2 s^{2} 2 p^{5} 3 d^{1} F_{3}$ \\
\hline & & & & & $28.0357^{\mathrm{e}}$ & $2 s^{2} 2 p^{5} 3 p^{3} D_{2}-2 s^{2} 2 p^{5} 3 d^{1} F_{3}$ \\
\hline $6^{\prime}$ & Fe XVII & $28.021 \pm 0.002$ & 28.0160 & 28.02 & 28.0274 & $2 s^{2} 2 p^{5} 3 p^{3} P_{2}-2 s^{2} 2 p^{5} 3 d^{3} D_{3}$ \\
\hline 7 & Fe XVII & $28.121 \pm 0.006$ & 28.1120 & 28.111 & 28.1288 & $2 s^{2} 2 p^{5} 3 p^{3} P_{1}-2 s^{2} 2 p^{5} 3 d^{3} D_{2}$ \\
\hline \multicolumn{7}{|c|}{${ }^{\mathrm{a}}$ Del Zanna and Ishikawa [24]. } \\
\hline \multicolumn{7}{|c|}{${ }^{\mathrm{b}}$ NIST Atomic Spectra Database, URL=http://www.nist.gov/pml/data/asd.cfm [35]. } \\
\hline \multicolumn{7}{|c|}{${ }^{\mathrm{c}}$ Theoretical calculations using the GRASP2K code by Jönssen et al. [26]. } \\
\hline & rors for the & $\begin{array}{l}\text { neasured waveleng } \\
\text { this transition is } \mathrm{n} \\
.41{ }^{3} \mathrm{D}_{2}+0.38{ }^{1} \mathrm{D}_{2}\end{array}$ & are due fr & $m$ fitting & $\begin{array}{l}\text { a Gaussia } \\
\text { n interactic } \\
\text { RASP2K o }\end{array}$ & $\begin{array}{l}\text { file. } \\
0.41{ }^{3} \mathrm{D}_{2}+0.39{ }^{1} \mathrm{D}_{2} \text { in the NIST } \\
\text { 26]. }\end{array}$ \\
\hline
\end{tabular}

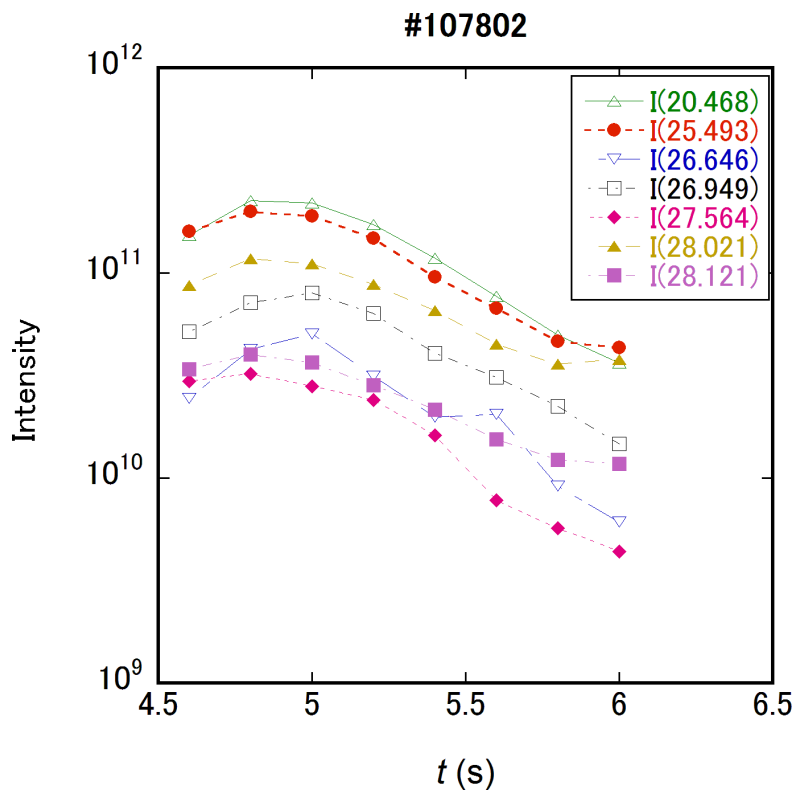

Fig. 4 Temporal evolution of Fe XVII line intensities for shot 107802.

coupling, so no LS terms were specified in the HULLAC calculation.

Figure 4 shows the temporal distribution for Fe XVII

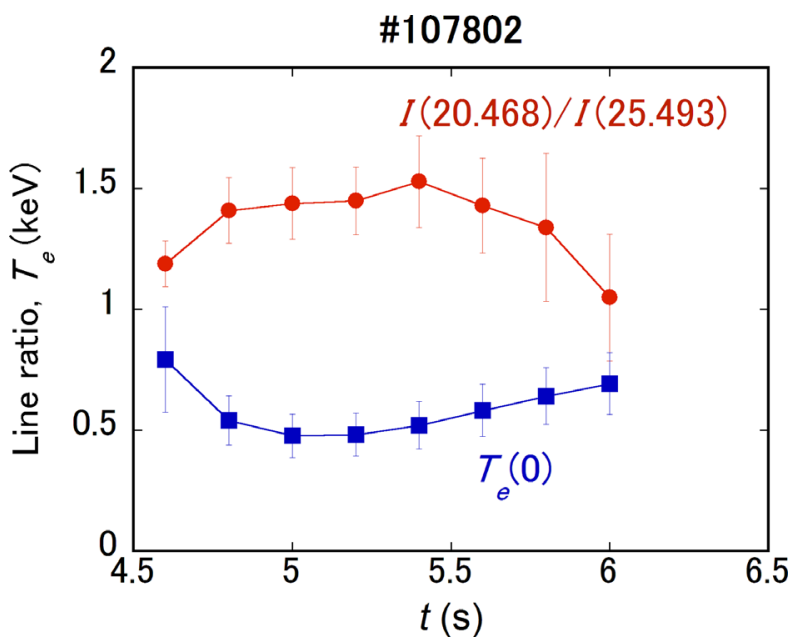

Fig. 5 Temporal evolution of Fe XVII line ratio for $\lambda 20.468 \mathrm{~nm}$ and $\lambda 25.493 \mathrm{~nm}$ (solid circles) and the central electron temperature (solid squares).

line photon intensities; all lines show the same evolution. Forty frames $(\Delta t=200 \mathrm{~ms})$ of the spectra were summed to determine line intensities by fitting to a Gaussian profile. Figure 5 shows the energy intensity ratio for Fe XVII 

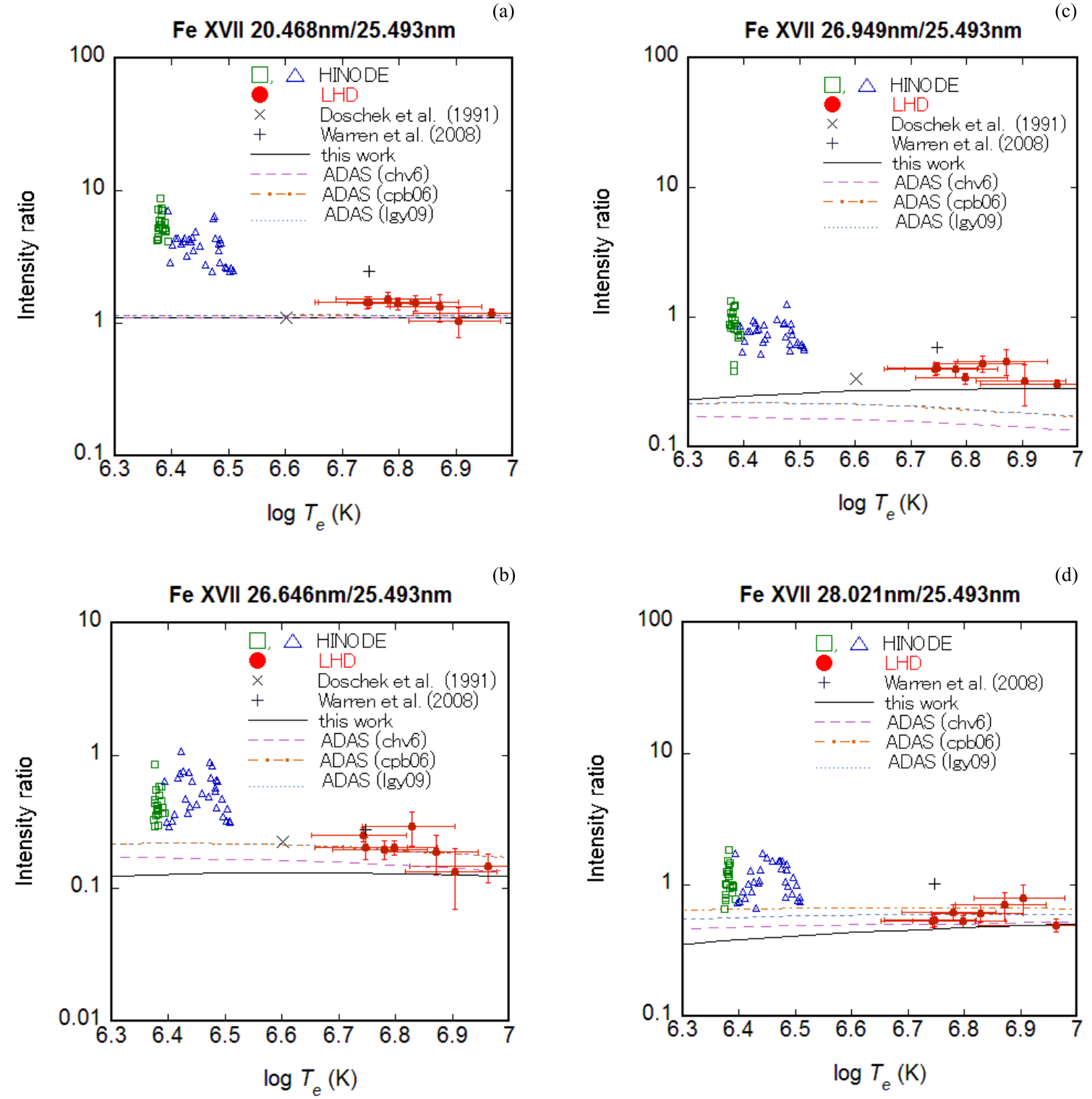

Fig. 6 Fe XVII line ratios as functions of electron temperature. (a) Fe XVII 20.468/25.493, (b) Fe XVII 26.646/25.493, (c) Fe XVII 26.949/25.493, and (d) Fe XVII 28.021/25.493. Solid dots with error bars are our measurements in LHD. Other measured values are from Hinode EIS measurements (open triangles and open squares [22] and plus symbol [21]) and the Skylab measurements (crosses [23]). Theoretical calculations are from our CR model (solid line), ADAS calculations with chv6 atomic dataset (dashed line), ADAS calculations with cpb06 atomic dataset (dot-dashed line), and ADAS calculations with lgy09 atomic dataset (dotted line).

20.468-nm and 25.493-nm lines and the central electron temperature as functions of time. Figure 6 (a) shows the measured energy intensity ratio as a function of central electron temperature. There are time variations in the intensity ratio, and the average intensity ratio is $1.35 \pm 0.16$, which is slightly larger than the theoretically obtained value of 1.1. The observed 20.468-nm line is possibly blended with Fe XIII 20.43-nm and 20.494-nm lines, as other Fe XIII lines such as the 20.383-nm line constantly appeared just after pellet injection until $t=6.4 \mathrm{~s}$ when the Fe XVII 20.468-nm line disappeared. Such lower charged Fe ions exist in the lower-temperature peripheral region, which intersects the line of sight. We need to subtract the contribution of these blended Fe XIII lines to estimate the correct Fe XVII 20.468-nm line intensity. Electron density and temperature of the Fe XIII emitting region were estimated using intensity ratios for Fe XII 18.66-nm and 19.51-nm lines, Fe XII 19.66-nm and Fe XIII $19.65 \mathrm{~nm}$ lines, and Fe XIII 20.38-nm and Fe XII 20.37-nm lines; the Fe XVII 20.468-nm line intensity was estimated by subtracting the intensities of the Fe XIII 20.43-nm and 20.49-nm lines. The corrected average intensity ratio becomes approximately 1.1 , which agrees well with the theoretical calculation [35]. Therefore, the branching ratio of Fe XVII 20.468 and $25.493 \mathrm{~nm}$, obtained by atomic physics, is confirmed by these LHD experiments. Details 
of the analysis for subtracting the contributions of Fe XIII lines are described by Watanabe et al. [36].

\section{Model Validation}

As described above, we confirm that the measured intensity ratio for Fe XVII 20.468 and $25.493 \mathrm{~nm}$ is consistent with the theoretical prediction. Now we compare measured energy intensity ratios for other Fe XVII lines listed in Table 1 with theoretical calculations by the CR model. Some of the results are shown in Fig. 6. Measured ratios are plotted as solid dots with error bars; other data measured for the solar plasma by Skylab [23] (crosses) and Hinode EIS [21, 22] (squares and triangles) are also plotted for comparison. In Fig. 6 (a), measured line ratios for 20.468 and $25.493 \mathrm{~nm}$ before the calibration are plotted. To compare with the measured 28.021-nm line intensity, the calculated intensities of two lines (nos. 6 and 6' in Table 1) were summed. Basically, the differences between the four theoretical calculations are mainly due to different atomic data, i.e., transition probabilities and electronimpact excitation effective collision strengths.

All measured ratios in the LHD plasma are roughly consistent with the calculated ratios, but ratios from Hinode EIS measurements are larger than the theoretical calculations, as seen in Fig. 6. Ratios from our CR model calculations are marginally consistent with the LHD measurements, but tend to be lower than the measurements. As described in Sec. 3, the measured ratios for $\lambda$ of 20.468 to $25.493 \mathrm{~nm}$ are slightly larger than the theoretical calculations (Fig. 6 (a)), because the $\lambda 20.468-\mathrm{nm}$ line is blended with the Fe XIII line. After subtracting the latter contribution, the ratio becomes consistent with the theoretical prediction. Similarly, our measured ratios for $\lambda$ of 26.949 to $25.493 \mathrm{~nm}$, whose average is $0.382 \pm 0.055$ (Fig. 6(c)), are marginally consistent with our CR model calculation but larger than the three ADAS calculations, perhaps because the $\lambda 26.949-\mathrm{nm}$ line is possibly blended with the Fe XIV 26.9357-nm line. As seen in Fig. 4, the line intensities of $\lambda 26.949$ and $26.646 \mathrm{~nm}$ increased at $t=4.8-5.0 \mathrm{~s}$, but other lines decreased. These behaviors of intensity also suggest contamination of other lines to $\lambda 26.949-\mathrm{nm}$ and $\lambda 26.646-\mathrm{nm}$ lines. The measured ratios for $\lambda 26.646-\mathrm{nm}$ to $25.493-\mathrm{nm}$ lines, whose average is $0.202 \pm 0.051$ (Fig. 6(b)), are roughly consistent with calculated ratios, but the candidate for line blending is the $\mathrm{Fe}$ XV 26.6377-nm line. The measured ratios for $\lambda$ of 28.021 to $25.493 \mathrm{~nm}$, whose average is $0.604 \pm 0.102$ (Fig. 6 (d)), show better agreement with all ADAS calculations than with our CR model calculation. The measured ratios for $\lambda$ of 27.564 to $25.493 \mathrm{~nm}$, whose average is $0.134 \pm 0.027$, and those for $\lambda 28.121-\mathrm{nm}$ to 25.493-nm lines, whose average is $0.202 \pm 0.028$ show tendencies similar to that of the ratio for $\lambda$ of 28.021 to $25.493 \mathrm{~nm}$ in Fig. 6 (d).

These results show that our CR model is marginally consistent with the LHD measurements for the Fe XVII line ratios. The ADAS calculations with the lgy09 atomic dataset show better agreement with the measurements than ours, except for the $\lambda$ 26.949-nm line. For constructing a better CR model, the lgy09 atomic dataset is therefore preferred. The reason is that the electron-impact excitation rate coefficient is calculated with the R-matrix method, which includes the resonance effect and is better than the distorted wave method in the lower collision-energy region, and many excited levels are considered. This atomic dataset can be obtained from the OPEN-ADAS website at http://open.adas.ac.uk/.

\section{Discussion}

The LHD measurements of the Fe XVII lines are almost consistent with the theoretical predictions from our CR model, as described in Sec. 4. However, Hinode EIS measurements still remain inconsistent with the theoretical predictions. For the $\lambda 20.468-n m$ line, Del Zanna and Ishikawa [24] suggested a blend with an unidentified line at $20.472 \mathrm{~nm}$, which showed the same morphology as the Fe VIII line in monochromatic images taken by Hinode EIS. This unidentified line could originate from the transition regions with lower temperatures at $T_{\mathrm{e}}=4-6 \times 10^{5} \mathrm{~K}$ (34-54 eV). For an active region core and a small flaring event on June 2, 2007, Watanabe et al. [35] reanalyzed the intensity ratio for $\lambda 20.468-\mathrm{nm}$ and $25.493-\mathrm{nm}$ lines by considering the contribution of the unidentified line originating from the transition region; the intensity ratio was significantly reduced to approximately $1.5-1.6$. Nevertheless, the ratio is higher than the theoretical prediction. This inconsistency indicates the need for re-examining the inflight intensity calibration of Hinode EIS instruments using two CCDs for the wavelength regions of 17-21 nm and $25-29 \mathrm{~nm}$ [37]. For the other five intensity ratios, the lines were measured with the same CCD and the inconsistency cannot be attributed to the intensity calibration of the instruments. The intensity for $\lambda$ of $25.493 \mathrm{~nm}$ seems systematically smaller than the theoretical prediction, but it is difficult to explain. In the future, we will need to examine Hinode EIS measurements in more detail.

\section{Summary}

We measured EUV spectra for LHD plasmas in which iron was injected by a TESPEL. NBI heating was well controlled, and we successfully produced plasmas with a central electron temperature of approximately $500 \mathrm{eV}$, which is suitable for producing Fe XVII ions. We detected seven Fe XVII lines whose wavelengths are consistent with Hinode EIS measurements, NIST database, and GRASP calculations. The measured intensity ratios for Fe XVII lines are consistent with the CR model calculations. Especially, the intensity ratio for Fe XVII 20.468-nm and 25.493-nm lines, after subtracting the effects of line blending, is consistent with the theoretical prediction of 1.1. This prediction was determined purely from the branching ratio due 
to the common upper level, although Warren et al. [21] reported a larger ratio from Hinode EIS measurements. The agreement of our LHD measurements and theoretical calculations for these ratios imply that the atomic data and the CR model are valid to be used for diagnostics. We suggest a preferred atomic dataset to obtain better agreement in the intensity ratios.

\section{Acknowledgments}

We acknowledge the LHD experimental group for their assistance. This work is partly supported by the NIFS Collaborative Research Programs (NIFS10KLPF008 and NIFS12KLPF027), the NINS Inter-institute collaborative program for Creation of New Research Area (Head Investigator: T. Watanabe), NIFS/NINS under the project of Formation of International Network for Scientific Collaborations (Head Investigator: H. Yamada). The authors would like to thank Enago (www.enago.jp) for the English language review.

[1] T. Kosugi et al., Solar Phys. 243, 3 (2007).

[2] J.L. Culhane et al., Solar Phys. 243, 19 (2007).

[3] J.A. Klimchuk, Solar Phys. 234, 41 (2006).

[4] A.K. Dupree et al. Astrophys. J. 299, L101 (1979).

[5] T. Kato et al., Astrophys. J. 492, 822 (1998).

[6] S. Imada et al., Astrophys. J. 742, 70 (2011).

[7] G.A. Doschek et al., Astrophys. J. 667, L109 (2007).

[8] S. Imada et al., Astrophys. J. 705, L208 (2009).

[9] N. Yamamoto et al., Astrophys. J. 689, 646 (2008).

[10] N. Yamamoto et al., J. Phys.: Conf. Ser. 163, 012023 (2009).

[11] T. Watanabe et al., Astrophys. J. 692, 1294 (2009).

[12] I. Murakami et al., Plasma Fusion Res. 5, S2021 (2010).
[13] H.A. Sakaue et al., J. Appl. Phys. 109, 073304 (2011).

[14] N. Nakamura et al., Astrophys. J. 739, 17 (2011).

[15] I. Skobelev et al., Astron. Astrophys. 511, A60 (2010).

[16] I. Murakami et al., accepted to IAEA Atomic and PlasmaMaterial Interaction Data for Fusion (2013).

[17] S. Sudo et al, Plasma Phys. Control. Fusion 44, 129 (2002).

[18] S. Sudo and N. Tamura, Rev. Sci. Instrum. 83, 023503 (2012).

[19] N. Nakamura et al., Rev. Sci. Instrum. 79, 063104 (2008).

[20] H.A. Sakaue et al., J. Instrumentation 5, C08010 (2010).

[21] H.P. Warren et al., Astrphys. J. 685, 1277 (2008).

[22] T. Watanabe et al., 38th COSPER-GA, E25-008-10 (oral presentation) (2010)

[23] G.A. Doscheck et al., Phys. Rev. A 43, 2565 (1991).

[24] G. Del Zanna and Y. Ishikawa, Astron. Astrophys. 508, 1517 (2009).

[25] P. Jönsson et al., Compt. Phys. Commun. 177, 597 (2007).

[26] P. Jönsson et al., Research Report of the National Institute for Fusion Science, NIFS-DATA-113 (2011); Atomic Data Nucl. Data Tables 100, 1 (2014).

[27] A. Bar-Shalon et al., J. Quant. Spect. Rad. Transf. 71, 179 (2001).

[28] H.P. Summers, The ADAS User Manual, version 2.6, http://www.adas.ac.uk (2004).

[29] K.P. Dere et al., Astron. Astrophys. 498, 915 (2009).

[30] S.D. Loch et al., J. Phys. B 39, 1 (2006).

[31] G.Y. Liang and N.R. Badnell, Astron. Astrophys. 518, A64 (2010).

[32] M.B. Chowduri et al., Rev. Sci. Instrum. 78, 023501 (2007).

[33] C. Dong et al., Rev. Sci. Instrum. 82, 113102 (2011).

[34] A. Kramida et al., URL=http://www.nist.gov/apml/data/ asd.cfm

[35] T. Watanabe et al., Plasma Fusion Res. 8, 2501105 (2013).

[36] T. Watanabe et al., in preparation (2014).

[37] G. Del Zanna, Astron. Astrophys. 555, A47 (2013). 